Leyton-Román, M.; Cordón, C.; Jiménez-Castuera, R. (2021) Analysis of Physical Activity and Lifestyles in University Students. Revista Internacional de Medicina y Ciencias de la Actividad $\begin{array}{lllllll}\text { Física } y & \text { el } & \text { Deporte } & \text { vol. } & 21 & \text { (81) } & \text { pp. }\end{array}$ Http://cdeporte.rediris.es/revista/revista81/artanalisis1228.htm

DOI: https://doi.org/10.15366/rimcafd2021.81.012

\title{
ORIGINAL
}

\section{ANÁLISIS DE LA ACTIVIDAD FÍSICA Y ESTILOS DE VIDA EN ESTUDIANTES UNIVERSITARIOS}

\section{ANALYSIS OF PHYSICAL ACTIVITY AND LIFESTYLES IN UNIVERSITY STUDENTS}

\author{
Leyton-Román, M. ${ }^{1}$; Cordón, C. $^{2}$ y Jiménez-Castuera, . $^{3}$ \\ ${ }^{1}$ Profesora Ayudante Doctora. Centro de Estudios del Deporte. Universidad Rey Juan Carlos de \\ Madrid (España) marta.leyton@uric.es \\ 2 Doctoranda. Facultad de Ciencias del Deporte. Universidad de Extremadura (España) \\ ccordong07@gmail.com \\ ${ }^{3}$ Profesora Titular en la Facultad de Ciencias del Deporte. Universidad de Extremadura (España) \\ ruthji@unex.es
}

Código UNESCO / UNESCO code: 3212 Salud Pública / Public Health, 3206. Clasificación Consejo de Europa / Council of Europe classification: 17. Otras: Actividad Física y Salud / Other: Physical Activity and Health

Recibido 7 de marzo de 2019 Received March 7, 2019

Aceptado 29 de junio de 2019 Accepted June 29, 2019

\section{RESUMEN}

El objetivo de este estudio fue analizar las relaciones entre las formas de motivación autodeterminadas, las necesidades psicológicas básicas y variables pertenecientes a estilos de vida saludables, así como hallar las diferencias en función del género, en estudiantes universitarios. Los cuestionarios validados se aplicaron a una muestra de 218 sujetos, 79 chicos y 139 chicas $(M=20,80$ años; $D T=2,12$ ). Los resultados mostraron que las formas de motivación más autodeterminadas se relacionaron de forma positiva con las necesidades psicológicas básicas, y con variables de estilo de vida que realzan la salud, y de manera negativa con el consumo de tabaco. Además, los chicos presentaron niveles más altos que las chicas en motivación intrínseca y extrínseca, necesidades psicológicas básicas, hábitos alimenticios, hábitos de descanso y práctica de actividad física. Se destaca la importancia de fomentar estrategias motivacionales para fomentar en los estudiantes un estilo de vida saludable.

PALABRAS CLAVE: Autodeterminación, necesidades psicológicas básicas, estilo de vida saludable, género. 


\section{ABSTRACT}

The purpose of this study was to analyze the relationships between the types of motivation, basic psychological needs and factors related to a healthy lifestyle, as well as finding the differences based on gender, in university students. The data was collected from a questionnaire given to a sample of 218 individuals, 79 men and 139 women between the ages of 18 and $32(M=20,80$ y $D T=2,12)$. The results show that the self-determined forms of motivation were significant, positively related with basic physiological needs, and with healthy lifestyles variables, but they were significant and negatively related with smoking habits. Additionally, men had a higher level in both intrinsic and extrinsic motivation compared to the women, as well as higher levels in basic psychological needs, eating habits, sleeping habits and physical activity. Encouraging motivational strategies to promote a healthy lifestyle in students is highlighted.

KEYWORDS: Self-determination, basic psychological needs, healthy lifestyle, gender. 


\section{INTRODUCCIÓN}

Dada la creciente preocupación por la adopción y el mantenimiento de un estilo de vida saludable, asociado a los importantes beneficios fisiológicos y psicológicos que conlleva la práctica de actividad física y al escaso interés que este hecho despierta entre los jóvenes y adultos una vez que han entrado en la etapa universitaria (García, García-Tapiero, y Ramos, 2012) se consideró oportuno realizar un análisis de la situación de los estudiantes universitarios.

Según Gallardo-Escudero, Muñoz, Planells y López (2015), la etapa universitaria implica una serie de cambios emocionales, fisiológicos y ambientales que conllevan unas costumbres y hábitos de riesgo donde, en muchos casos, se mantendrán a lo largo de la vida. Estos cambios condicionan los hábitos de alimentación, práctica de actividad física y otras variables, que pueden suponer un riesgo para la salud (Santinelli, 2011). Por tanto, si se quiere promocionar y educar para la salud, se debe tener presente los estilos de vida llevados a cabo por las personas (Leyton, Batista, Lobato, Aspano y Jiménez, 2017).

Consiguientemente, en el presente estudio se analizaron las principales variables que forman parte de los estilos de vida saludables: consumo de tabaco, consumo de alcohol, consumo de otras drogas, hábitos de descanso, hábitos alimenticios (alimentación equilibrada y respeto al horario de las comidas) y práctica de actividad física (Pastor, Balaguer y García-Merita, 1998).

La base sobre la que se apoya este trabajo es la Teoría de la Autodeterminación (Deci y Ryan, 1985) y el modelo jerárquico de la motivación (Vallerand, 1997).

Una de las teorías que ayuda a explicar la motivación de los estudiantes universitarios es la Teoría de la Autodeterminación (Decy y Ryan, 1985; Ryan y Deci, 2000). La Teoría de la Autodeterminación analiza el grado en que las personas realizan sus acciones de forma voluntaria, por propia elección (Carratalá, 2004). Esta propone que la motivación se encuentra enmarcada a lo largo de un continuo en el que se distinguen tres niveles (Vansteenkiste, Lens y Deci, 2006; Vansteenkiste, Niemiec y Soenens, 2010): motivación intrínseca (la más autodeterminada, supone realizar una actividad por el propio placer que supone practicarla), motivación extrínseca (realizar una actividad por recompensa o reconocimientos externos a la propia actividad), dentro de la cual se distinguen de mayor a menor autodeterminación, la regulación integrada, la conducta se realiza libremente y la integración ocurre cuando la persona evalúa la conducta y actúa en congruencia con sus valores y sus necesidades, la regulación identificada, donde la conducta es altamente valorada y el individuo la juzga como importante por lo que la realizará libremente aunque la actividad no sea agradable, la regulación introyectada, donde los motivos de participación en una actividad son principalmente el reconocimiento social, las presiones internas o los sentimientos de culpa y, la regulación externa, donde la conducta es realizada por la existencia de premios o recompensas; $y$ desmotivación o falta de intención de actuar, la forma de motivación menos autodeterminada (Decy y Ryan, 2000). 
Partiendo de esta teoría, Vallerand (1997), desarrolló el modelo jerárquico de la motivación, relacionando diferentes constructos a partir de tres niveles: el nivel global, referido a la motivación que habitualmente está presente en la vida de las personas, el nivel contextual, que se da en un contexto concreto, y el nivel situacional, referido a la motivación que una persona posee en un momento y en una actividad específica. Centramos el presente estudio en el nivel contextual.

Según Deci y Ryan (1991), la motivación es determinada por factores sociales, como la práctica de actividad física, que influye en el comportamiento de las personas y es motivada por tres necesidades psicológicas básicas (NPB) primarias y universales: autonomía, comprendida por los esfuerzos de las personas por sentirse el origen de sus acciones y determinar su propio comportamiento; competencia, que se basa en tratar de controlar el resultado y experimentar eficacia; y relación con los demás, que hace referencia al esfuerzo por relacionarse y sentir una relación auténtica con los demás. La satisfacción de las NPB producirá un aumento de las formas de motivación más autodeterminadas, originando consecuencias positivas para la práctica deportiva a nivel cognitivo, emocional y comportamental. Una de las posibles consecuencias analizadas en el presente estudio es el mantenimiento de un estilo de vida saludable, que supone la persistencia en la práctica de actividad física y unos hábitos alimenticios adecuados, así como la evitación de sustancias nocivas (Deci y Ryan, 2000).

Por tanto, es importante conocer cómo se pueden desarrollar las formas de motivación más autodeterminadas, ya que hay estudios que demuestran su relación con una serie de consecuencias motivacionales positivas, como la intención de seguir practicando actividad física o la satisfacción de las NPB (Franco, Coterón, Martínez y Brito, 2017; Leyton et al., 2017; Sweet, Fortier y Blanchard, 2014) y, por otro lado, la relación de factores motivacionales negativos, como la desmotivación, asociado al menor compromiso deportivo o a estilos de vida menos saludables (García-Calvo, Sánchez, Leo, Sánchez y Amado, 2011; Jiménez, Cervelló, García, Santos y Iglesias, 2007; MorenoMurcia, González-Cutre y Cervelló, 2008).

Teniendo en cuenta las diferencias de género, y de acuerdo con la bibliografía consultada, se ha demostrado que los hombres valoran más los beneficios externos a la práctica deportiva (Moreno-Murcia, López, Alonso, MartínezGalindo y González-Cutre, 2007) que las mujeres, encontrándose así más intrínsecamente motivadas (Recours, Souville y Griffet, 2004). Por otra parte, algunos autores como Moreno-Murcia, Cano, González-Cutre, Cervelló y Ruiz (2009) encontraron una mayor desmotivación a favor del género masculino. En relación a las NPB, un estudio realizado por Amado, Sánchez- Miguel, Leo, Sánchez-Oliva, y García-Calvo (2014), demuestra que para el género masculino la variable que más predice la percepción de utilidad del deporte es la satisfacción de la necesidad de relaciones sociales mientras que para el género femenino es la necesidad de autonomía.

En cuanto al mantenimiento de un estilo de vida saludable, los hombres valoran más los beneficios externos a la práctica deportiva y son físicamente más activos (Ballesteros, Freidin, Krause y Borda, 2016; González y Portolés, 2016; López, 
Calabuig y Pérez, 2013; Moreno-Murcia, López, Alonso, Martínez- Galindo y González-Cutre, 2007). A diferencia de las chicas, cuidan más la alimentación y consumen menos alcohol, tabaco y otras drogas (Castillo, Balaguer y GarcíaMérita, 2007; Delgado, Bautista, Inglés, Espada y Torregrosa, 2005; GallardoEscudero et al., 2015; Pamies, Quiles y Quiles, 2012).

El presente estudio tuvo como objetivo principal analizar las relaciones existentes entre las formas de motivación autodeterminadas, el grado de satisfacción de las NPB y diferentes variables pertenecientes a estilos de vida saludables, así como hallar las diferencias con respecto al género en estudiantes universitarios formulando como hipótesis:

1. Las formas de motivación más autodeterminadas se relacionarán de forma positiva y significativa con las NPB, los hábitos alimenticios, los hábitos de descanso y la práctica de actividad física.

2. Las formas de motivación más autodeterminadas se relacionarán de forma negativa con aquellas variables que son nocivas para la salud (consumo de tabaco y alcohol).

3. Los chicos mostrarán mayores valores que las chicas en motivación intrínseca y extrínseca, NPB, hábitos alimenticios, hábitos de descanso y práctica de actividad física.

\section{MÉTODO}

El estudio recibió la aprobación de la Comisión de Bioética y Bioseguridad de la Universidad de Extremadura (España) siguiente la línea de la Declaración de Helsinki. Todos los participantes fueron tratados de acuerdo con las pautas éticas de la American Psychological Association con respecto al consentimiento del participante, el consentimiento de los padres / tutores, la confidencialidad y el anonimato.

\section{Diseño}

El estudio llevado a cabo, se encuadra dentro de los estudios descriptivos, cuantitativos y de corte transversal en poblaciones mediante encuestas (Montero y León, 2007).

\section{Participantes}

La muestra de este estudio perteneció a una población de estudiantes universitarios, más concretamente al alumnado de $1^{\circ}$ y $4^{\circ}$ del grado de Ciencias del Deporte, Veterinaria y Educación Social. Para realizar la selección de la muestra utilizó un procedimiento de muestreo intencional o de conveniencia por conglomerados atendiendo a la titulación y al curso, en un total de 6 clases con 25-40 alumnos en cada una de ellas. 
La muestra estaba compuesta por 218 sujetos de ambos géneros (79 chicos y 139 chicas) con edades comprendidas entre 18 y 28 años $(M=20.80$ y $D T=$ 2,12). De todos ellos, 77 pertenecían a los grados de Ciencias del Deporte (49 chicos y 28 chicas), 80 de Veterinaria (23 chicos y 57 chicas) y 61 de Educación Social (7 chicos y 54 chicas).

\section{Procedimiento}

En primer lugar, se contactó con los docentes de las diferentes facultades explicándoles los objetivos de la investigación y pidiéndoles permiso para que nos dejasen los últimos 20 minutos de sus respectivas clases para que los alumnos pudiesen completar los cuestionarios previstos. A continuación, se concretaron varios días y horas para la administración de los mismos.

Tras la aceptación por parte de los profesores, y tras haber cumplimentado un consentimiento informado, los estudiantes realizaron los cuestionarios en su aula de manera individual bajo la presencia de un investigador y de sus respectivos docentes en un clima que les permitió concentrarse sin distracciones de ningún tipo. Previamente a la aplicación de los cuestionarios, se realizó una presentación a los estudiantes con el objetivo del estudio, dándoles instrucciones para su cumplimentación. Se hizo hincapié en que contestaran con absoluta sinceridad, ya que los resultados eran confidenciales y los cuestionarios se cumplimentaban de manera anónima. El tiempo empleado dependió del ritmo de cada clase pero, aproximadamente, fueron 20 minutos.

\section{Variables e instrumentos de medida}

Con el objetivo de medir las formas de motivación autodeterminadas, se aplicó el Cuestionario de la Regulación Conductual en el Deporte (Behavioral Regulation in Sport Questionnaire: BRSQ) de Lonsdale, Hodge y Rose (2008) validado al contexto español por Moreno-Murcia, Marzo, Martínez-Galindo y Conte (2011) compuesto por 36 ítems, seis para evaluar cada una de las formas de motivación: motivación intrínseca (e.g. "Porque disfruto cuando intento alcanzar metas a largo plazo"), regulación integrada (e.g. "Porque es parte de lo que soy"), regulación identificada (e.g. "Porque los beneficios del deporte son importantes para mi"), regulación introyectada (e.g. "Porque me sentiría avergonzado si lo abandono"), regulación externa (e.g. "Porque otros me presionan") y desmotivación (e.g. "No sé por qué lo hago"). La sentencia previa fue "Participo en este deporte...".

Con el propósito de medir la satisfacción de las NPB, se utilizó la Psychological Need Satisfaction in Exercise Scale (PNSE) de Wilson, Rogers, Rodgers y Wild (2006), validada al contexto español por Moreno-Murcia et al. (2011). El cuestionario está formado por 18 ítems, seis para evaluar cada una de las necesidades: competencia (e.g. "Tengo confianza para hacer los ejercicios más desafiantes", autonomía (e.g. "Creo que puedo tomar decisiones en mis clases") y relación con los demás (e.g. "Me siento unido a mis compañeros porque me aceptan como soy"). La sentencia previa fue "En mis entrenamientos...". 
Con la finalidad de medir los hábitos de estilo de vida saludables, se utilizó el Cuestionario de Estilos de Vida Saludable (EVS) de Leyton, Lobato, Batista, Aspano y Jiménez (2018). Consta de 12 ítems divididos en los siguientes factores: hábitos de descanso (e.g. "Respeto los horarios de descanso"), consumo de Tabaco (e.g. "Me siento bien cuando fumo"), alimentación equilibrada (e.g. "Considero que tengo una alimentación equilibrada y sana") y respeto al horario de las comidas (e.g. "Siempre desayuno"). Para la medición de las variables Alcohol y Drogas, se utilizó la versión de Jiménez, Cervelló, García-Calvo, Santos-Rosa y Del Villar (2006), donde se utilizaron los factores consumo de alcohol (e.g. "A veces tomo alcohol incluso los días de diario"), y consumo de drogas (e.g. "Me resulta fácil conseguir drogas").

Finalmente, la variable practica de actividad física, se midió a través del International Physical Activity Questionnaire (IPAQ) de Craig et al. (2003), validada al contexto español por Roman et al. (2010). Se utilizó la versión corta (7 ítems) con preguntas que hacen referencia al tiempo que los alumnos destinaban a estar físicamente activos en los últimos 7 días (e.g. "Durante los últimos 7 días ¿Cuánto tiempo pasó sentado en un día hábil?"). Según Mantilla y Gómez (2007), el cuestionario evalúa tres características específicas de actividad: 1 (baja): no registran actividad física o no alcanza el nivel medio, 2 (media): 3 o más días de actividad física vigorosa por lo menos 20 min por día, 5 o más días de actividad física moderada o caminar por lo menos $30 \mathrm{~min}$, o 5 o más días de cualquier combinación de actividad física leve, moderada o vigorosa y, por último, 3 (alta): 3 o más días de actividad física vigorosa más de 30 minutos por día o 7 o más días de cualquier combinación de actividad física leve, moderada o vigorosa.

\section{Análisis de los datos}

Se realizó un análisis de fiabilidad para comprobar la consistencia interna de los cuestionarios y creamos las diferentes variables. Se realizaron pruebas de normalidad, con el objetivo de determinar qué tipo de estadística se debía emplear. Las medidas de asimetría, curtosis, Kolmogorov-Smirnov con la corrección de Lilliefors verificaron que la distribución de la muestra era normal, por lo que se aplicó la estadística paramétrica. Posteriormente, se realizó un análisis descriptivo, un análisis de correlación y un análisis de varianza. Todo esto se llevó a cabo con el programa estadístico SPSS 18.

\section{RESULTADOS}

\section{Análisis descriptivo y de fiabilidad}

En la Tabla 1, aparecen los valores descriptivos (media y desviación típica) y de fiabilidad de cada una de las variables de la investigación. 
Rev.int.med.cienc.act.fís.deporte - vol. 21 - número 81 - ISSN: 1577-0354

Tabla 1. Estadísticos descriptivos y análisis de fiabilidad de las variables medidas a través de los cuestionarios.

\begin{tabular}{|c|c|c|c|c|}
\hline Variables & Rango & Media & D.T. & Alpha de Cronbach \\
\hline M.I.General & $1-7$ & 5,08 & 1,92 & 0,93 \\
\hline M.I. hacia el conocimiento & $1-7$ & 4,35 & 2,00 & 0,93 \\
\hline M.I. hacia la ejecución & $1-7$ & 5,01 & 1,78 & 0,91 \\
\hline M.I. hacia la estimulación & $1-7$ & 4,81 & 1,86 & 0,92 \\
\hline Regulación integrada & $1-7$ & 4,17 & 2,01 & 0,93 \\
\hline Regulación identificada & $1-7$ & 4,61 & 1,73 & 0,84 \\
\hline Regulación Introyectada & $1-7$ & 2,45 & 1,45 & 0,80 \\
\hline Regulación externa & $1-7$ & 1,75 & 1,14 & 0,79 \\
\hline Desmotivación & $1-7$ & 1,89 & 1,31 & 0,79 \\
\hline NPB Competencia & $1-7$ & 4,87 & 1,71 & 0,94 \\
\hline NPB Autonomía & $1-7$ & 4,67 & 1,61 & 0,90 \\
\hline NPB Relación con los demás & $1-7$ & 4,04 & 1,67 & 0,89 \\
\hline Consumo de tabaco & $1-7$ & 1,95 & 1,54 & 0,89 \\
\hline Consumo de alcohol & $1-7$ & 2,78 & 1,46 & 0,83 \\
\hline Consumo de otras drogas & $1-7$ & 2,36 & 1,42 & 0,74 \\
\hline Hábitos de descanso & $1-7$ & 4,20 & 1,56 & 0,79 \\
\hline Alimentación equilibrada & $1-7$ & 4,19 & 1,38 & 0,72 \\
\hline Respeto al horario de comidas & $1-7$ & 4,71 & 1,48 & 0,80 \\
\hline Practica de actividad física & $1-3$ & 1,97 & 0,94 & - \\
\hline
\end{tabular}

Nota. M.I. = Motivación intrínseca; NPB= Necesidades Psicológicas Básicas; DT = Desviación Típica.

Se puede observar que la motivación intrínseca general $(5,08)$ y la motivación intrínseca hacia la ejecución $(5,01)$ presentan el mayor promedio en la muestra. Las medias más bajas se encontraron en la regulación externa $(1,75)$ y en la desmotivación $(1,89)$. Además, se analizó el método de consistencia interna a través del alpha de Cronbach. Según Steiner (2003), el valor mínimo aceptable para el coeficiente alfa de Cronbach es 0,70 y por debajo de ese valor la consistencia interna de la escala utilizada es baja, habiendo una excepción en el factor "práctica de actividad física", que no presentó dicho alpha ya que los ítems que componen esta variable no están correlacionados entre sí.

\section{Análisis de correlaciones}




\section{Rev.int.med.cienc.act.fís.deporte - vol. 21 - número 81 - ISSN: 1577-0354}

Tabla 2. Análisis de correlación.

\begin{tabular}{|c|c|c|c|c|c|c|c|c|c|c|c|c|c|c|c|c|c|c|c|}
\hline Variables & 1 & 2 & 3 & 4 & 5 & 6 & 7 & 8 & 9 & 10 & 11 & 12 & 13 & 14 & 15 & 16 & 17 & 18 & 19 \\
\hline 1. MI General & - & & & & & & & & & & & & & & & & & & \\
\hline 2. MI Conocimiento & $0,83^{\star \star}$ & - & & & & & & & & & & & & & & & & & \\
\hline 3. MI Ejecución & $0,81^{* *}$ & $0,81^{* *}$ & - & & & & & & & & & & & & & & & & \\
\hline 4. MI Estimulación & $0,87^{* *}$ & $0,85^{\star *}$ & $0,88^{* *}$ & - & & & & & & & & & & & & & & & \\
\hline 5. R. Integrada & $0,78^{\star *}$ & $0,86^{* *}$ & $0,80^{\star *}$ & $0,85^{* *}$ & - & & & & & & & & & & & & & & \\
\hline 6. R. Identificada & $0,75^{\star *}$ & $0,79^{\star *}$ & $0,82^{\star \star}$ & $0,79^{\star *}$ & $0,79^{\star \star}$ & - & & & & & & & & & & & & & \\
\hline 7. R. Introyectada & $0,17^{*}$ & $0,31^{* *}$ & $0,38^{\star *}$ & $0,31^{* *}$ & $0,43^{\star *}$ & $0,41^{\star *}$ & - & & & & & & & & & & & & \\
\hline 8. R. Externa & $-0,14^{*}$ & 0,04 & 0,00 & $-0,02$ & 0,07 & 0,03 & $0,60^{\star \star}$ & - & & & & & & & & & & & \\
\hline 9. Desmotivación & $-0,16^{*}$ & $-0,10$ & $-0,13^{*}$ & $-0,14$ & 0,08 & $0,20^{\star *}$ & $0,19^{\star \star}$ & $0,53^{\star \star}$ & '- & & & & & & & & & & \\
\hline 10. NPB Competencia & $0,75^{* \star}$ & $0,71^{\star *}$ & $0,78^{\star *}$ & $0,75^{\star *}$ & $0,75^{\star \star}$ & $0,72^{\star *}$ & $0,30^{\star *}$ & 0,00 & $-0,12$ & - & & & & & & & & & \\
\hline 11. NPB Autonomía & $0,56^{* *}$ & $0,50^{* *}$ & $0,61^{* *}$ & $0,56^{* *}$ & $0,52^{\star *}$ & $0,54^{* *}$ & $0,24^{* *}$ & 0,04 & 0,00 & $0,73^{\star \star}$ & - & & & & & & & & \\
\hline 12. NPB Relación & $0,62^{\star \star}$ & $0,68^{\star *}$ & $0,65^{\star \star}$ & $0,66^{\star \star}$ & $0,68^{\star \star}$ & $0.64^{\star *}$ & $0,36^{\star \star}$ & $0,23^{\star *}$ & 0,02 & $0,73^{\star \star}$ & $0,50^{\star *}$ & - & & & & & & & \\
\hline 13. Tabaco & $-0,18^{\star *}$ & $-0,12$ & $-0,14^{*}$ & $-0,16^{*}$ & $-0,14^{*}$ & $-0,15^{*}$ & 0,02 & $0,23^{\star *}$ & $0,25^{* \star}$ & $-0,13$ & $-0,04$ & $-0,11$ & - & & & & & & \\
\hline 14. Alcohol & $-0,07$ & $-0,12$ & $-0,06$ & $-0,07$ & $-0,13$ & $-0,15^{*}$ & 0,09 & $0,27^{* *}$ & $0,38^{\star *}$ & $-0,10$ & $-0,00$ & 0,06 & $0,35^{\star *}$ & - & & & & & \\
\hline 15. Otras Drogas & 0,03 & $-0,01$ & 0,05 & 0,04 & 0,00 & $-0,02$ & $0,24^{\star \star}$ & $0,32^{* *}$ & $0,33^{* \star}$ & 0,01 & $0,17^{*}$ & 0,02 & $0,51^{\star *}$ & $0,48^{\star \star}$ & - & & & & \\
\hline 16. Descanso & $0,27^{* *}$ & $0,25^{* *}$ & $0,24^{\star *}$ & $0,25^{* *}$ & $0,26^{\star \star}$ & $0,28^{* *}$ & $0,19^{\star \star}$ & 0,02 & $-0,05$ & $0,30^{* *}$ & $0,20^{* *}$ & $0,30^{* *}$ & $-0,15^{\star}$ & $-0,04$ & $-0,07$ & - & & & \\
\hline 17. Alimentación & $0,45^{\star *}$ & $0,41^{* *}$ & $0,43^{* *}$ & $0,42^{* *}$ & $0,41^{* *}$ & $0,44^{* *}$ & $0,25^{\star \star}$ & 0,06 & 0,07 & $0,44^{\star *}$ & $0,42^{* *}$ & $0,32^{\star *}$ & $-0,07$ & 0,00 & 0,09 & $0,39^{* *}$ & - & & \\
\hline 18. H. Comidas & $0,36^{\star *}$ & $0,33^{* *}$ & $0,37^{\star \star}$ & $0,36^{\star *}$ & $0,36^{\star \star}$ & $0,38^{\star *}$ & $0,18^{\star \star}$ & 0,04 & $-0,04$ & $0,41^{\star *}$ & $0,35^{\star *}$ & $0,33^{* \star}$ & $-0,19^{\star \star}$ & $-0,09$ & $-0,05$ & $0,53^{* \star}$ & $0,56^{\star \star}$ & - & \\
\hline 19. Practica AF & $0,41^{* \star}$ & $0,43^{\star *}$ & $0,41^{* *}$ & $0,43^{\star *}$ & $0,46^{\star *}$ & $0,38^{\star *}$ & $0,14^{*}$ & 0,01 & $-0,06$ & $0,44^{\star \star}$ & $0,25^{\star *}$ & $0,37^{\star \star}$ & $-0,02$ & $-0,12$ & $-0,02$ & $0,14^{*}$ & $0,15^{\star}$ & 0,10 & - \\
\hline
\end{tabular}

Nota. MI = Motivación Intrínseca; R. = Regulación; NPB= Necesidad Psicológica Básica; H.=Horario; AF=Actividad física. ${ }^{\star *}$. La correlación es significativa al nivel 0,01. *. La correlación es significativa al nivel 0,05 . 
Se llevó a cabo el análisis de correlaciones bivariadas con el objetivo de ver las relaciones establecidas entre las variables que conforman el estudio (Tabla 2). Los resultados mostraron que la motivación intrínseca general se relacionó positiva y significativamente con la motivación intrínseca hacia el conocimiento, la ejecución y la estimulación, la regulación integrada, identificada e introyectada, la necesidad psicológica básica de competencia, autonomía y de relación con los demás, los hábitos de descanso, los hábitos alimenticios y la práctica de actividad física. $Y$ de forma negativa y significativa con la regulación externa, la desmotivación y el consumo de tabaco. La motivación intrínseca hacia el conocimiento se asoció positiva y significativamente con la motivación intrínseca general, hacia la ejecución, la estimulación, la regulación integrada, identificada e introyectada, la necesidad psicológica básica de autonomía, competencia y relación con los demás, los hábitos de descanso, los hábitos alimenticios y la práctica de actividad física. La motivación intrínseca hacia la ejecución se relacionó positiva y significativamente con la motivación intrínseca general, hacia el conocimiento, la estimulación, la regulación integrada, identificada e introyectada, la necesidad psicológica básica de competencia, autonomía y relación con los demás, los hábitos de descanso, los hábitos alimenticios y la práctica de actividad física $y$, de manera negativa y significativa, con la desmotivación y el consumo de tabaco. Por último, la motivación intrínseca hacia la estimulación se asoció positiva y significativamente con la motivación general, la regulación integrada, identificada e introyectada, la necesidad psicológica básica de competencia, autonomía y relación con los demás, los hábitos de descanso, los hábitos alimenticios y la práctica de actividad física. $Y$ negativa y significativamente con el consumo de tabaco. Finalmente, la desmotivación se asoció de manera positiva y significativa con la regulación identificada, introyectada, externa, el consumo de tabaco, alcohol y otras drogas y, de manera negativa y significativa, con la motivación intrínseca general y la ejecución.

\section{Análisis de las diferencias en función del género}

Se utilizó un análisis ANOVA en función del género como variable independiente, siendo las variables dependientes las analizadas en el presente estudio (Tabla 3 ). Se puede observar que todas las medias estaban a favor de los varones, exceptuando la variable de desmotivación.

Las variables en las que se hallaron diferencias significativas fueron la motivación intrínseca general $(0,00)$, la motivación intrínseca hacia el conocimiento $(0,00)$, hacia la ejecución $(0,01)$, hacia la estimulación $(0,00)$, la regulación integrada $(0,00)$, la regulación identificada $(0,00)$, la regulación introyectada $(0,02)$, la competencia $(0,00)$, la autonomía $(0,00)$ y las relaciones con los demás $(0,00)$, así como el consumo de drogas $(0,03)$ y la práctica de actividad física $(0,01)$.

Concretar que la diferencia se encontró en la motivación intrínseca general presentada en los alumnos-as, donde los chicos $(M=5,80)$ presentaron más motivación que las chicas $(M=4,65)$. Los resultados del análisis también mostraron que son los chicos $(M=5,23)$ quienes presentan una mayor motivación intrínseca hacia el conocimiento, que las chicas $(M=3,83)$. 
En relación a la motivación intrínseca hacia la ejecución, los chicos $(M=5,45)$ presentaron más media que las chicas $(M=4,75)$. Lo mismo ocurrió con la motivación intrínseca hacia la estimulación, donde se halló que los chicos $(M=$ $5,38)$ tienen más que las chicas $(M=4,48)$.

Con respecto a la regulación integrada, se observó que los chicos $(M=4,93)$ poseen más que las chicas $(M=3,71)$. Lo mismo ocurrió con la regulación identificada, donde fue mayor en los chicos $(M=5,10)$ más que en las chicas $(M$ $=4,33$ ) y, en la regulación introyectada, con una media de 2,76 en chicos y 2,27 en chicas.

Por otra parte, en cuanto a la necesidad psicológica básica de competencia, se observó una mayor media en los chicos $(M=5,50)$, que en las chicas $(M=4,50)$. Al igual que en el resto de NPB, donde en la autonomía los chicos $(M=5,11)$ mostraron más autonomía que las chicas $(M=4,40)$, manteniendo estos también más media en la necesidad psicológica básica de relaciones con los demás ( $M$ $=4,69)$ en relación con chicas $(M=3,67)$. 
Tabla 3. Análisis de varianza en función del género

\begin{tabular}{|c|c|c|c|c|c|}
\hline Variables & $\begin{array}{c}\text { Media } \\
\text { Cuadrática }\end{array}$ & $F$ & $p$ & $\begin{array}{l}\text { Media } \\
\text { chicos }\end{array}$ & $\begin{array}{l}\text { Media } \\
\text { chicas }\end{array}$ \\
\hline MI General & 66,87 & 19,76 & 0,00 & 5,80 & 4,65 \\
\hline $\begin{array}{c}\text { MI hacia el } \\
\text { conocimiento }\end{array}$ & 99,69 & 28,06 & 0,00 & 5,24 & 3,84 \\
\hline $\begin{array}{l}\text { MI hacia la } \\
\text { ejecución }\end{array}$ & 24,25 & 7,93 & 0,01 & 5,45 & 4,76 \\
\hline $\begin{array}{l}\text { MI hacia la } \\
\text { estimulación }\end{array}$ & 40,69 & 12,43 & 0,00 & 5,38 & 4,49 \\
\hline $\begin{array}{l}\text { Regulación } \\
\text { integrada }\end{array}$ & 74,78 & 20,13 & 0,00 & 4,93 & 3,72 \\
\hline $\begin{array}{l}\text { Regulación } \\
\text { identificada }\end{array}$ & 30,14 & 10,51 & 0,00 & 5,10 & 4,33 \\
\hline $\begin{array}{l}\text { Regulación } \\
\text { Introyectada }\end{array}$ & 12,40 & 6,06 & 0,02 & 2,77 & 2,27 \\
\hline $\begin{array}{l}\text { Regulación } \\
\text { Externa }\end{array}$ & 3,41 & 2,62 & 0,11 & 1,92 & 1,66 \\
\hline Desmotivación & 0,32 & 0,18 & 0,67 & 1,84 & 1,92 \\
\hline $\begin{array}{c}\text { NPB } \\
\text { Competencia }\end{array}$ & 51,12 & 19,00 & 0,00 & 5,51 & 4,50 \\
\hline NPB Autonomía & 22,48 & 10,20 & 0,00 & 5,12 & 4,41 \\
\hline $\begin{array}{l}\text { NPB Relación } \\
\text { con los demás }\end{array}$ & 52,54 & 20,58 & 0,00 & 4,69 & 3,67 \\
\hline $\begin{array}{c}\text { Consumo de } \\
\text { tabaco }\end{array}$ & 0,26 & 0,11 & 0,74 & 1,91 & 1,98 \\
\hline $\begin{array}{c}\text { Consumo de } \\
\text { alcohol }\end{array}$ & 0,62 & 0,29 & 0,59 & 2,85 & 2,74 \\
\hline $\begin{array}{l}\text { Consumo de } \\
\text { otras drogas }\end{array}$ & 9,91 & 5,02 & 0,03 & 2,64 & 2,20 \\
\hline $\begin{array}{c}\text { Hábitos de } \\
\text { descanso }\end{array}$ & 2,33 & 0,96 & 0,33 & 4.34 & 4.12 \\
\hline $\begin{array}{c}\text { Alimentación } \\
\text { equilibrada }\end{array}$ & 3,76 & 1,99 & 0,16 & 4,36 & 4,09 \\
\hline $\begin{array}{l}\text { Respeto al } \\
\text { horario de } \\
\text { comidas }\end{array}$ & 1,72 & 0,78 & 0,38 & 4,83 & 4,65 \\
\hline $\begin{array}{c}\text { Practica de } \\
\text { Actividad Física }\end{array}$ & 6,52 & 7,55 & 0,01 & 2,20 & 1,84 \\
\hline
\end{tabular}

Nota. Ml = Motivación intrínseca; NPB= Necesidades Psicológicas Básicas; $p=$ significación $>0,05$

Este estudio demostró también que los chicos $(M=2,63)$ consumen más drogas que las chicas $(M=2,19)$, al igual que son ellos $(M=2,20)$ quienes realizan más actividad física que las chicas $(M=1,84)$. 


\section{DISCUSIÓN}

Dada la relevancia que tiene analizar los procesos motivacionales y los estilos de vida en los estudiantes universitarios, se procede a discutir los resultados mostrados anteriormente, en función de las hipótesis planteadas.

Teniendo en cuenta la primera hipótesis: "Las formas de motivación más autodeterminadas se relacionarán de forma positiva y significativa con las NPB, los hábitos alimenticios, los hábitos de descanso y la práctica de actividad física", debemos aclarar que los resultados obtenidos la confirman. Al igual que en la presente investigación, numerosos estudios han demostrado esta relación (Balaguer, Castillo y Duda, 2008; Franco et al., 2017; Moreno-Murcia, Conte, Borges y González-Cutre, 2008; Samperio, Jiménez, Lobato, Leyton y Claver, 2016; Standage, Duda y Ntoumanis, 2006). Destacar que la necesidad psicológica básica de relaciones sociales obtuvo valores más bajos en comparación con el resto de NPB. Esto puede ser debido a los valores más altos de autonomía que presentan, pues comienzan a ser más independientes dentro de un ambiente completamente diferente a lo vivido en la etapa escolar.

Ha sido demostrada también la relación positiva entre las formas de motivación más autodeterminadas y los hábitos alimenticios, los hábitos de descanso y la práctica de actividad física (Cascaes et al., 2017; Jiménez, et al., 2007; MorenoMurcia et al., 2008; Varela y Silvestre, 2010). Por lo tanto, es lógico que los estudiantes que presenten más motivación autodeterminada mantengan hábitos de vida más saludables, interviniendo directamente en su desarrollo físico, psíquico y mental en el periodo universitario.

En relación a la práctica de actividad física, relaciones similares fueron encontradas en un estudio reciente donde relacionaron la motivación y la práctica de actividad física a través del IPAQ, en estudiantes universitarios. Práxedes, Sevil, Moreno, Del Villar y García-González (2016), determinaron la importancia de desarrollar procesos motivacionales para mejorar los niveles de actividad física, sobre todo en titulaciones menos relacionadas con la misma.

El presente estudio recalca la importancia de desarrollar la satisfacción de las NPB (Deci y Ryan, 2000), permitiendo la propia elección de las actividades a los estudiantes, estableciendo objetivos que supongan un reto alcanzable, promoviendo las relaciones sociales, proporcionando un feedback positivo e individualizado, incrementando así la motivación autodeterminada para conseguir un mayor compromiso con un estilo de vida más saludable.

En relación con la segunda hipótesis: "Las formas de motivación más autodeterminadas se relacionarán de forma negativa con aquellas variables que son nocivas para la salud (consumo de tabaco y alcohol) ", se pude observar que los resultados también la confirman. Álvarez- Aguirre, Alonso-Castillo y Guidorizzi (2014) y Moreno et al. (2008), afirmaron que los estudiantes que presentan mayor probabilidad de consumo de drogas como el alcohol y tabaco son los que están expuestos a diferentes factores de riesgo personales, del contexto universitario en el que éstos se desarrollan y de las cogniciones 
relacionadas con los estilos de vida saludables, relacionados con una menor motivación autodeterminada.

Carballo et al. (2013), reafirman que el periodo universitario es una etapa donde los estudiantes empiezan a iniciarse en el consumo de sustancias nocivas, especialmente al salir de fiesta con sus iguales. En este sentido, Martínez y Villar (2004) y Ruiz-Ruiseño et al. (2011) coinciden en que el "botellón" es un contexto que favorece el consumo de alcohol y tabaco (Cortés, Espejo y Giménez, 2008), así como las relaciones sociales entre los jóvenes.

En relación a la tercera hipótesis planteada: 'Los chicos mostrarán mayores valores que las chicas en motivación intrínseca y extrínseca, NPB, hábitos alimenticios, hábitos de descanso y práctica de actividad física", diversos estudios la confirman (Brunet y Sabiston, 2009; Moreno-Murcia et al., 2009). En el presente trabajo, fue la necesidad psicológica básica de competencia la que presenta mayores valores. Esto nos indica que los hombres se sienten más competentes a la hora de practicar deporte que las mujeres, lo cual puede ser debido a que se ven con más cualidades para superar cualquier reto motriz que se le plantee, pues tradicionalmente las situaciones deportivas han estado más adaptadas a la capacidad de los hombres que de las mujeres (Moreno-Murcia et al., 2009). Por otra parte, Según Romero-Martín, Gelpi-Fleta, Mateu-Serra y Lavega-Burgués (2017), en un estudio con estudiantes universitarios, determinaron que los chicos tenían una mayor experiencia emocional en las actividades físicas, que las chicas, destacando también la importancia que tenía el tipo de actividad para predecir la significatividad de la experiencia emocional.

Es por esto que existe la necesidad de reconstruir el deporte actual desde una perspectiva que profundice en los valores democráticos alejados de los estereotipos sexistas (Moreno, Cervelló y González-Cutre, 2006).

Por otro lado, la principal limitación del trabajo ha sido el hecho de haber empleado únicamente cuestionarios, ya que los datos obtenidos no proporcionan tanta información como si se hubiera llevado a cabo una triangulación de métodos tanto desde el punto de vista de las perspectivas (profesores, padres, entrenadores..) como de las fuentes (hojas de registro, entrevistas...) combinando este tipo de investigaciones con otras de corte más observacional, considerando también otra limitación el hecho de que en la muestra utilizada no había el mismo número de chicas que de chicos, lo que puede incidir sobre los resultados, pero esto se corresponde con la cifra real de estudiantes que se encontraban en ese momento en las aulas, por lo que sería conveniente para futuros estudios realizar un estudio longitudinal, donde se pueda analizar a todos los alumnos pertenecientes a ese curso y no sólo a los que se encuentran en el aula en ese momento, permitiendo así una ampliación de la muestra.

A partir del presente estudio, creemos necesaria la creación por parte de las diferentes universidades, de un ambiente importante para intervenciones destinadas a ayudar a los adolescentes en el mantenimiento de un estilo de vida saludable, así como la satisfacción de las necesidades psicológicas básicas de competencia, autonomía y relaciones con los demás, teniendo también importancia el conocimiento, por parte de los órganos representativos de las 
universidades, de las preferencias deportivas de los estudiantes para poder fomentar el gusto por la práctica de actividad física. Por tanto, es necesario flexibilizar la oferta deportiva existente para que el nivel de adhesión a las actividades deportivas aumente en la comunidad universitaria. Por último, sería interesante realizar investigaciones posteriores centradas únicamente en aquellos sujetos que presenten menos motivación autodeterminada con el fin de satisfacer las NPB que estén frustradas, y favorecer hábitos saludables entre los mismos.

\section{CONCLUSIONES Y APLICACIONES PRÁCTICAS}

Una vez llevado a cabo el estudio, se sacaron las siguientes conclusiones:

- Las formas de motivación más autodeterminadas se relacionaron de forma positiva y significativa con las NPB, los hábitos alimenticios, los hábitos de descanso y la práctica de actividad física, y de forma negativa con las variables nocivas para la salud (consumo de tabaco y alcohol).

Por tanto, como aplicación práctica sería interesante que desde el entorno universitario se fomentara la práctica de actividad física a través de actividades formativas extraescolares, haciendo hincapié en las relaciones entre universitarios y ya que nos resulta muy importante el componente social en cualquier actividad deportiva, con el fin de crear mayor adherencia a la práctica y a unos estilos de vida más saludables, sugiriendo el fomento de la actividad física a través de actividades formativas creadas desde la universidad que sustituyan gran parte del tiempo empleado en dichas salidas, y disminuyan el consumo de tabaco y alcohol fomentando las relaciones sociales y los estilos de vida saludables que se prolonguen en el tiempo. De este modo se previenen enfermedades y problemas metabólicos, cardiovasculares, respiratorios, hormonales y psicológicos, entre otros (Lindsay et al., 2014).

- En relación al género, los chicos mostraron mayores valores que las chicas en motivación intrínseca y extrínseca, NPB, hábitos alimenticios, hábitos de descanso y práctica de actividad física.

Una solución práctica para esto sería favorecer la actividad deportiva como medio complementario en el contexto universitario, siendo las chicas quienes también se encarguen de planificar distintos eventos celebrados en las facultades, como el aumento de fruta en cafeterías, actividades con contenido formativo de EVS, gincanas con pruebas físicas, etc.. favoreciendo así un estilo de vida saludable y permitiendo a todos los individuos conocerse mejor, expresarse, adquirir habilidades y demostrar sus capacidades, sirviendo de estímulo a la incorporación de la mujer a una sociedad sin distinción de género potenciando su participación en la elaboración y concreción de políticas deportivas que aún es escasa comparada con otros ámbitos (Lanzuela, Matute, Tifner, Gallizo y Gil- Lacruz, 2007).

Por lo tanto, se considera de especial importancia fomentar la satisfacción de las NPB (competencia, autonomía y relación con los demás), lo que aumentaría las formas de motivación más autodeterminadas, y en consecuencia la obtención de 
estilos de vida saludables de los estudiantes universitarios, a través del fomento de la práctica de actividad física, una mejora de los hábitos alimenticios, hábitos de descanso y evitación del consumo de sustancias nocivas para la salud. 


\section{REFERENCIAS BIBLIOGRÁFICAS}

Álvarez-Aguirre, A., Alonso-Castillo, M. M. y Guidorizzi, A. C. (2014). Factores predictivos del uso de alcohol y tabaco en los adolescentes. Revista latinoamericana de Enfermagem, 22(6), 1056-1062.

https://doi.org/10.1590/0104-1169.3570.2516

Amado, D., Sánchez-Miguel, P. A., Leo, F. M., Sánchez-Oliva, D. y García-Calvo, T. (2014). Diferencias de género en la motivación y percepción de utilidad del deporte escolar. Revista Internacional de Medicina y Ciencias de la Actividad Física y el Deporte, 14 (56), 651-664.

Balaguer, I., Castillo, I. y Duda, J. (2008). Apoyo a la autonomía, satisfacción de las necesidades, motivación y bienestar en deportistas de competición: un análisis de la teoría de la autodeterminación. Revista de Psicología del Deporte, 17(1), 123-139.

Ballesteros, M., Freidin, B., Krause, M. y Borda, P. (2016). La práctica de actividad física entre varones y mujeres de clase media en el AMBA: motivaciones, percepción de beneficios y limitaciones para su continuidad. Educación Física y Ciencia, 18(2), 1-20.

Brunet, J. y Sabinton, C. M. (2009). Social physique anxiety and physical activity: A self-determination theory perspective. Psychology of Sport and Exercise, 10(3), 329-335. https://doi.org/10.1016/j.psychsport.2008.11.002

Carballo, J. L., Marín, M., Jáuregui, V., García, G., Espada, J., Orgilés, M. y Piqueras, J.A. (2013). Consumo excesivo de alcohol y rendimiento cognitivo en estudiantes de secundaria de la provincia de Alicante. Health and Addictions: Salud y drogas, 13(2), 157-163.

https://doi.org/10.21134/haaj.v13i2.205

Carratalá, E. (2004). Análisis de la teoría de las metas de logro y de la autodeterminación en los planes de especialización deportiva de la Generalitat Valenciana. (Tesis doctoral). Universidad de Valencia, Valencia.

Cascaes, F., Da Rosa, R., Valdivia, B.,Barbosa, P., Da Silva, R., Oliveira M. y Pereira, A. (2017). Ejercicio físico, calidad de vida y salud de diabéticos tipo 2. Revista de Psicología del Deporte, 26(1), 13-25.

Castillo, I., Balaguer, I. y García- Mérita, M. (2007). Efecto de la práctica de actividad física y de la participación deportiva sobre el estilo de vida saludable en la adolescencia en función del género. Revista de Psicología del Deporte, 16(2), 201-210.

Cortés, M. T., Espejo, B. y Giménez, J. A. (2008). Aspectos cognitivos relacionados con la práctica del botellón. Psicothema, 20(3), 396-402.

Craig, C. L., Marshall, A. L., Sjostrom, M., Bauman, A. E., Booth, M. L. y Ainsworth, B. E. (2003). International physical activity questionnaire: 12country reliability and validity. Medicine and Science in Sports and Exercise, 35, 1381-95. https://doi.org/10.1249/01.MSS.0000078924.61453.FB

Deci, E. L. y Ryan, R. M. (1985). The general casuality orientations scale: Selfdetermination in personality. Journal of Research in Personality, 19, 109134. https://doi.org/10.1016/0092-6566(85)90023-6

Deci, E. L. y Ryan, R. M. (1991). A motivational approach to self: Integration in personality. En Nebraska symposium on motivation (Ed.), Perspectives on motivation (pp. 237-288). Lincoln, NE: University of Nebraska Press. 
Deci, E. L. y Ryan, R. M. (2000). The 'what' and 'why' of goal pursuits: human needs and the self-determination of behavior. Psychoanalytic Inquiry, 11, 227-68. https://doi.org/10.1207/S15327965PLI1104 01

Delgado, B., Bautista, R., Inglés, C. J., Espada, J. P., Torregrosa, M. S. y GarcíaFernández, J. M. (2005). Diferencias de género en el consumo de alcohol y tabaco de estudiantes de educación secundaria obligatoria. Salud y Drogas, 5(2), 55-65. https://doi.org/10.21134/haaj.v5i2.110

Franco, E., Coterón, J., Martínez, H. E. y Brito, J. (2017). Perfiles motivacionales en estudiantes de educación física de tres países y su relación con la actividad física. Suma Psicológica, 24(1), 18. https://doi.org/10.1016/j.sumpsi.2016.07.001

Gallardo-Escudero, A., Muñoz, M. J., Planells, E. M. y López, I. (2015). La etapa universitaria no favorece el estilo de vida saludable en las estudiantes granadinas. Nutrición Hospitalaria, 31(2), 975-979.

García, D. G., García-Tapiero, G. P. y Ramos, D. M. (2012). Determinantes de los estilos de vida y su implicación en la salud de jóvenes universitarios. Hacia la Promoción de la Salud, 17(2), 169- 185.

García-Calvo, T., Sánchez, P. A., Leo, F. M., Sánchez, D. y Amado, D. (2011). Incidencia de la Teoría de Autodeterminación sobre la persistencia deportiva. Revista Internacional de Ciencias del Deporte, 25(7), 266-276. https://doi.org/10.5232/ricyde2011.02502

González, J. y Portolés, A. (2016). Recomendaciones de actividad física y su relación con el rendimiento académico en adolescentes de la Región de Murcia. Retos, 29, 100-104. https://doi.org/10.47197/retos.v0i29.39936

Jiménez, R., Cervelló, E., García, T., Santos, F. J. y Iglesias, D. (2007). Estudio de las relaciones entre motivación, práctica deportiva extraescolar y hábitos alimenticios y de descanso en estudiantes de Educación Física. International Journal of Clinical and Health Psychology, 7(2), 385401.

Jiménez, R., Cervelló, E. M, García-Calvo, T., Santos-Rosa, F. J., y Del Villar, F. (2006). Relaciones entre las metas de logro, la percepción del clima motivacional, la valoración de la Educación Física, la práctica deportiva extraescolar y el consumo de drogas en estudiantes de Educación Física. Revista Mexicana de Psicología, 23(2), 253-265.

Lanzuela, Y., Matute, S., Tifner, S., Gallizo, M. E. y Gil- Lacruz, M. (2007). Sedentarismo y percepción de la salud: Diferencias de género en una muestra aragonesa. Revista Internacional de Medicina y Ciencias de la Actividad Física y el Deporte, 7(28), 344-358.

Leyton, M., Batista, M., Lobato, S., Aspano, M. I. y Jiménez, R. (2017). Application of two intervention programs in order to optimize motivation and to improve eating habits in adult and elderly women. Journal of human kinetics, 59(1), 131-142. https://doi.org/10.1515/hukin-2017-0153

Leyton, M., Lobato, S., Batista, M., Aspano, M. I. y Jiménez, R. (2018). Validación del cuestionario de estilo de vida saludable (EVS) en una población española. Revista Iberoamericana de Psicología del Ejercicio y el Deporte, 13(1), 23-31.

Lindsay, A. R., Hongu, N., Spears, K., Idris, R., Dyrek, A. y Manore, M. M. (2014). Field assessments for obesity prevention in children and adults: physical 
activity, fitness, and body composition. Journal of Nutrition Education and Behavior. 46(1), 43-53. https://doi.org/10.1016/j.jneb.2013.03.013

Lonsdale, C., Hodge, K. y Rose, E. (2008). The behavioral regulation in sport questionnaire (BRSQ): Instrument development and initial validity evidence. Journal of Sport \& Exercise Psychology, 30(3), 323-355. https://doi.org/10.1123/jsep.30.3.323

López, E., Calabuig, F. y Pérez, C. (2013). Relación entre la actividad física, los hábitos alimentarios y el consumo y actitud hacia las drogas en un instituto valenciano. Edetania, 44, 219-229.

Mantilla, S. C. y Gómez, A. (2007). El cuestionario internacional de actividad física. Un instrumento adecuado en el seguimiento de la actividad física poblacional. Revista Iberoamericana de Fisioterapia y Kinesiología, 10(1), 48-52. https://doi.org/10.1016/S1138-6045(07)73665-1

Martínez, G. y Villar, M. (2004). Estudio descriptivo del uso de drogas en adolescentes de Educación Media Superior de Ciudad de Monterrey N.L. Revista Latinoamericana Enfermagem, 12, 391 -397. https://doi.org/10.1590/S0104-11692004000700014

Montero, I. y León, O. G. (2007). Guía para nombrar los estudios de investigación en Psicología. International Journal of Clinical and Health Psychology, 7, 847-862.

Moreno-Murcia, J. A., Cano, F., González-Cutre, D., Cervelló, E. y Ruiz, L. M. (2009). Flow disposicional en salvamento deportivo: Una aproximación desde la teoría de la autodeterminación. Revista de Psicología del Deporte, 18, 23-35.

Moreno-Murcia, J. A., Cervelló, E. y González-Cutre, D. (2006). Motivación autodeterminada y flujo disposicional en el deporte. Anales de Psicología, 22(2), 310- 317.

Moreno-Murcia, J. A., Conte, L., Borges, F. y González- Cutre, D. (2008). Necesidades psicológicas básicas, motivación intrínseca y propensión a la experiencia autotélica en el ejercicio físico. Revista Mexicana de Psicología, 25(2), 305-312.

Moreno-Murcia, J. A., González-Cutre, D. y Cervelló, E. (2008). Motivación y salud en la práctica físico-deportiva: Diferencias según el consumo de alcohol y tabaco. International Journal of Clinical and Health Psychology, 8(2), 483-494.

Moreno-Murcia, J. A., López, M., Martínez-Galindo, C., Alonso, N. y GonzálezCutre, D. (2007). Efectos del género, la edad y la frecuencia de práctica en la motivación y disfrute del ejercicio físico. Fitness and Performance Journal, 6(3), 140-146.

Moreno-Murcia, J. A., Marzo, J. C., Martínez-Galindo, C. y Marín, L. C. (2011). Validación de la escala de "Satisfacción de las necesidades psicológicas básicas" y del cuestionario de la "Regulación conductual del deporte" al contexto español. RICYDE. Revista Internacional de Ciencias del Deporte, 7(26), 355-369. https://doi.org/10.5232/ricyde2011.02602

Pamies, L., Quiles, Y. y Quiles, M. J. (2012). Diferencias de género y edad en los hábitos alimentarios y sobrepeso de los adolescentes españoles. Revista Española de Nutrición Comunitaria, 18(1), 19-26. 
Pastor, Y., Balaguer, I. y García-Merita, M. (1998). Una revisión sobre las variables de estilos de vida saludables. Revista de Psicología de la Salud, 10(1), 15-52.

Práxedes, A., Sevil, J., Moreno, A., Del Villar, F. y García-González, L. (2016). Niveles de actividad física y motivación en estudiantes universitarios. diferencias en función del perfil académico vinculado a la práctica físicodeportiva. Journal of Sport and Health Research, 8(3), 191-204.

Recours, R. A., Souville, M. y Griffet, J. (2004). Expressed motives for informal and club/association based sports participation. Journal of Leisure Research, 36, 1-22. https://doi.org/10.1080/00222216.2004.11950008

Roman, B., Serra, L., Hagströmer, M., Ribas, L., Sjöström, M. y Segura, R. (2010). International Physical Activity Questionnaire: reliability and validity in a Spanish population. European Journal of Sport Science, 10(5), 297304. https://doi.org/10.1080/17461390903426667

Romero-Martín, M. R., Gelpi-Fleta, P., Mateu-Serra, M. y Lavega-Burgués, P. (2017). Influencia de las prácticas motrices sobre el estado emocional de estudiantes universitarios/Influence of Motor Practices on University Students' Emotional State. Revista Internacional de Medicina y Ciencias de la Actividad Física y del Deporte, 17(67), 449-466. https://doi.org/10.15366/rimcafd2017.67.004

Ruiz-Ruiseño, J., Ruiz-Juan, F. y Zamarripa, J. I. (2011). Alcohol y tabaco en adolescentes españoles y mexicanos y su relación con la actividad físicodeportiva y la familia. Revista Panamericana de Salud Pública, 31(3), 211220. https://doi.org/10.1590/S1020-49892012000300005

Ryan, R. M. y Deci, E. L. (2000). Self-determination theory and the facilitation of intrinsic motivation, social development, and well-being. The American Psychologist, 55, 68-78. https://doi.org/10.1037/0003-066X.55.1.68

Samperio, J., Jiménez, R., Lobato, S., Leyton, M. y Claver, F. (2016). Variables motivacionales predictoras de las barreras para la práctica de ejercicio físico en adolescentes. Cuadernos de Psicología del Deporte, 16(2), 65-76.

Santinelli, M. P. (2011). Hábitos alimentarios y estilo de vida en adolescentes de 13 a 17 años de edad, que concurren a la escuela secundaria de Pavón Arriba, de la provincia de Santa Fe. (Tesis doctoral). Universidad abierta interamericana, Rosario, Argentina.

Standage, M., Duda, J. L. y Ntoumanis, N. (2006). Student's motivational processes and their relationship to teacher ratings in school physical education: A self-determination theory approach. Research Quarterly for Exercise and Sport, 77, 100-110.

https://doi.org/10.1080/02701367.2006.10599336

Steiner, D. (2003). Starting at the beginning: an introduction to coefficient alpha and internal consistency. Journal of Personality Assessment, 80(1), 99-103. https://doi.org/10.1207/S15327752JPA8001 18

Sweet, S. N., Fortier, M. S. y Blanchard, C. M. (2014). Investigating motivational regulations and physical activity over 25 weeks. Journal of Physical Activity and Health, 11(5), 1052-1056. https://doi.org/10.1123/jpah.2012-0057

Vallerand, R. J. (1997). Toward a hierarchical model of intrinsic and extrinsic motivation. (pp. 271-360). Academic Press: New York.

https://doi.org/10.1016/S0065-2601(08)60019-2 
Vansteenkiste, M., Lens, W. y Deci, E. L. (2006). Intrinsic versus extrinsic goal contents in self-determination theory: Another look at the quality of academic motivation. Educational Psychology, 41(1), 19-31. Doi:10.1207/s15326985ep4101 4.

https://doi.org/10.1207/s15326985ep41014

Vansteenkiste, M., Niemiec, C. y Soenens, B. (2010). The development of the five mini-theories of self-determination theory: An historical overview, emerging trends, and future directions. In T. Urdan \& S. Karabenick (Eds.). Advances in Motivation and Achievement, vol. 16: The decade ahead (pp.105-166). Bingley, UK: Emerald. https://doi.org/10.1108/S07497423(2010)000016A007

Varela, G. y Silvestre, D. (2010). Nutrición, vida activa y deporte. España: IM \& C.

Wilson, P. M., Rogers, W. T., Rodgers, W. M. y Wild, T. C. (2006). The psychological need satisfaction in exercise scale. Journal of Sport and Exercise Psychology, 28(3), 231-251. https://doi.org/10.1123/jsep.28.3.231

Referencias totales / Total references: $55(100 \%)$

Referencias propias de la revista/Journal's own references: $3(5,5 \%)$

Rev.int.med.cienc.act.fís.deporte - vol. 21 - número 81 - ISSN: 1577-0354 